Document downloaded from:

http://hdl.handle.net/10251/65356

This paper must be cited as:

Belenguer Martínez, A.; Borja, A.; Díaz Caballero, E.; Esteban González, H.; Boria Esbert, VE. (2015). Systematic Procedure to Avoid Unintended Polarity Mismatch in the Cascade Connection of Multiport Devices with Symmetric Feeding Lines. IET Microwaves Antennas and Propagation. 9(11):1128-1135. doi:10.1049/iet-map.2014.0167.

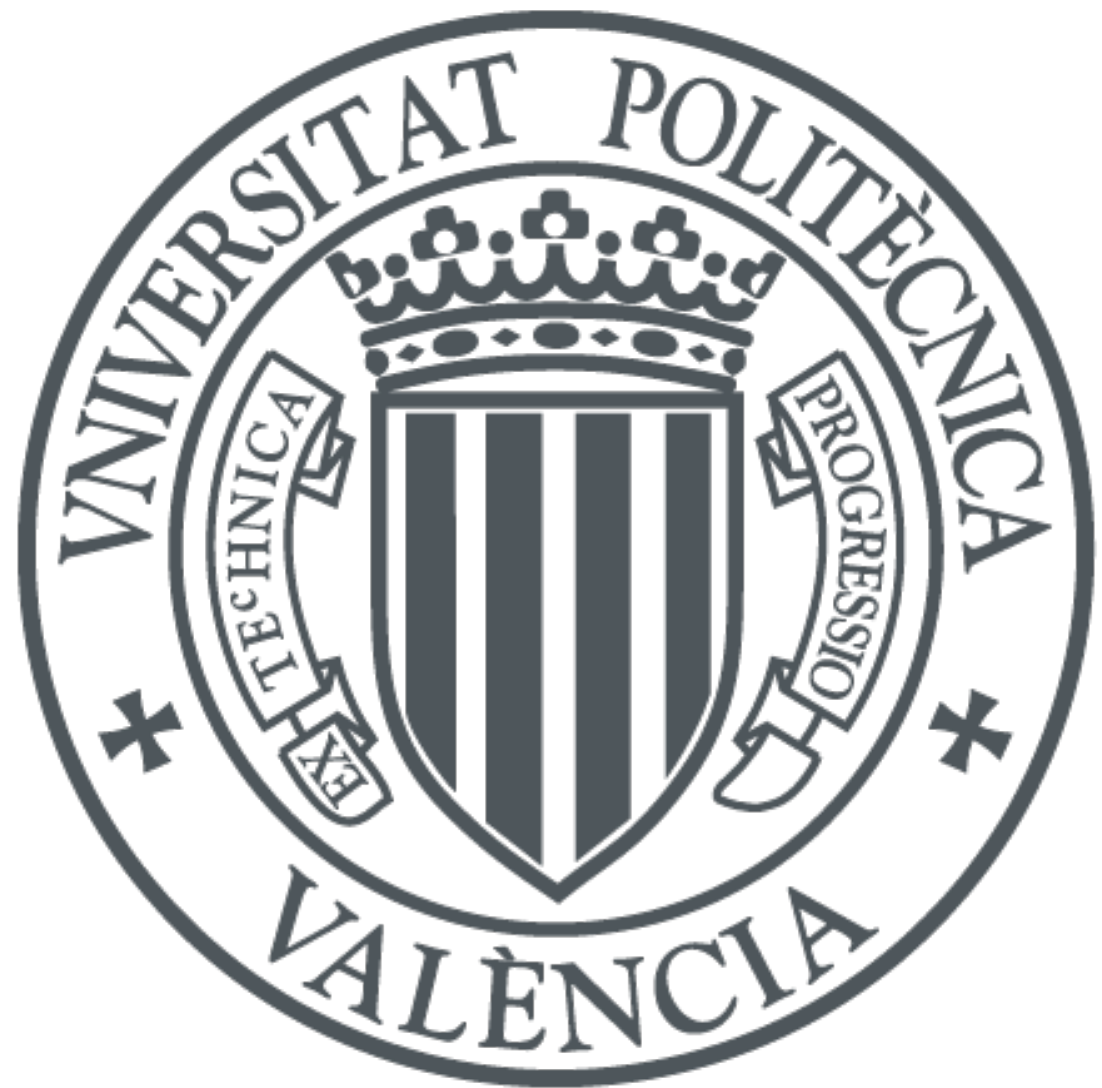

The final publication is available at

http://dx.doi.org/10.1049/iet-map.2014.0167

Copyright Institution of Engineering and Technology (IET)

Additional Information

This paper is a postprint of a paper submitted to and accepted for publication in [journal] and is subject to Institution of Engineering and Technology Copyright. The copy of record is available at IET Digital Library 


\section{Systematic procedure to avoid unintended polarity mismatch in the cascade connection of multiport devices with symmetric feeding lines}

ISSN 1751-8725

Received on 20th March 2014 Revised on 11th December 2014 Accepted on 12th March 2015 doi: 10.1049/iet-map.2014.0167 www.ietdl.org

\section{Angel Belenguer ${ }^{凶}$, Alejandro Lucas Borja ${ }^{2}$, Elena Diaz Caballero ${ }^{3}$, Hector Esteban ${ }^{4}$,} Vicente E. Boria 4

${ }^{1}$ Ingenieria Electrica, Electronica, Automatica y Comunicaciones, Universidad de Castilla-La Mancha, Campus universitario, Cuenca, Spain ${ }^{2}$ Departamento de Ingeniería Eléctrica, Electrónica, Automática y Comunicaciones, Universidad de Castilla-La Mancha, Campus Universitario, Cuenca, Spain

${ }^{3}$ Universitat Politecnica de Valencia, Valencia, Spain

${ }^{4}$ Departamento de Comunicaciones, Universidad Politecnica de Valencia, Valencia, Spain

$凶$ E-mail: angel.belenguer@uclm.es

Q2 Abstract: The traditional cascading of generalised scattering matrices (GSMs) approach assumes that the modal sets at the connected ports of a cascaded network are strictly equal. This implies a careful selection of the modal polarities (numerical port solution), or the reference systems (analytical port solution), of every port to be connected. Usually, the connection scheme of every device is known a priori, during the solving stage. Then, the individual GSMs are preprocessed, or auxiliary devices intended to correct possible modal mismatches at the ports are included in appropriate positions among the cascade, so that the traditional cascading-by-pairs approach can be directly applied. This scheme clearly complicates the reutilisation of previously calculated GSMs, and mixes the cascading with the solution of the individual building blocks. In this study, a systematic procedure is proposed to define the polarity of the modes at

30 the ports of a multiport device fed with transmission lines or waveguides showing a single or double symmetry. The modified expressions to calculate the scattering parameters of the cascade of two multiport devices, incorporating the appropriate regular modal corrections to apply when this criterion is used to define the modal polarity at the ports, is also presented in this study. This strategy is more convenient from the point of view of programming, less error-prone and easier to implement.

\section{Introduction}

The cascade connection of generalised scattering matrices (GSMs) $[1,2]$ was first studied in the late 50's and early 60's. First, the cascade of two two-port devices was solved and, at the same time, Redheffer [3] developed an special matrix product (in non-standard notation), known as 'star' product, which can be

45 used to cascade the GSMs of two $2 \mathrm{~N}$-port devices connected through $N$ ports. Next, Kaplan and Stock $[4,5]$ demonstrated that this 'star' product was useful to solve the general case, that is, the cascade connection of two devices with an arbitrary number of ports, for example, $N$ and $M$, and also an arbitrary number of nections, for example, $K$. There are several strategies to deduce the expressions of the cascaded GSM. For example, one can force the equality between emergent and incident waves at the connected ports [6]. Therefore all of these expressions for the cascade connection of GSMs are valid if, and only if, the modal sets belonging to a pair of connected ports are actually identical.

55 To ensure this equality, for an asymmetric transmission line or waveguide, it is sufficient to appropriately define the mode polarity. Unfortunately, when the line is symmetric it is more difficult to ensure the coincidence of the connected modal sets. This fact is explained in detail in the following sections.

To ensure the coincidence of the connected modal sets of symmetric transmission lines or waveguides, it is sufficient to ensure that the reference systems used to define the modes of every pair of connected ports coincide, or, equivalently, that the integration lines used to define the polarity of the modes overlap when the ports are connected. The definition of these reference

65 coordinate systems, or integration lines, is trivial if a cascade of two-port devices is performed. Unfortunately, this is not the case for more complex cascading schemes, involving devices with three or more ports. Even so, it is, indeed, possible to define an appropriate set of reference systems (or modal polarities) for every port of a given device, but only if its connection arrangement is known a priori. Unfortunately, in this case, the GSM of this device cannot be directly used to characterise this same device in a different position in the cascade, if its connection scheme is different. To reutilise the already calculated GSM, its scattering parameters must be appropriately corrected. It is also possible to insert an auxiliary device between the non-coincident connected ports in order to adapt possible modal mismatches. Both solutions are valid and possible, but hard to implement because the modal correction applied to a device will depend on its connection scheme.

Recently, in [7], a very efficient strategy to connect multiport devices has been proposed. This strategy, which is based on an iterative Krylov's solver, is able to provide, not only the response of the cascaded network at the free ports, but also the modal weights at the internal ports. When this technique was elaborated, the problems regarding the connection of devices with symmetric feeding lines were detected, and a correction was proposed. This problem arises when multimodal scattering matrices are cascaded and it is assumed that all reflected waves are exactly equal to the same order incident modes of the connected contiguous device. This may not be true for some non-symmetric modes if the coordinate systems of both ports are not identical. In [7], however, Q3 only one of the symmetries of a rectangular waveguide was considered (the symmetry of a rectangular waveguide is double). Besides, the partial correction of [7] was applied to the Krylov's-based technique, and not to the traditional cascading 
expressions. Therefore in this paper, a rigorous study of possible modal mismatches at the connected ports is presented, and a systematic criterion to define the polarity of the modes (or the reference systems of the ports) is proposed for general lines or waveguides with a single or double transversal symmetry. Consequently, the traditional expressions for cascading the GSMs of two networks are extended to correct all of these possible modal mismatches. The coplanar waveguide (CPW) line, the microstrip line, the coplanar strips line, the rectangular waveguide with a single ridge and the grounded CPW are examples of transmission lines, or waveguides, with a single transversal symmetry. On the other hand, the rectangular waveguide is an example of waveguide with double symmetry. A regular criterion for the modal polarity (or reference system) election simplifies the analysis tool used to obtain the independent GSMs of the building blocks, because it can be configured regardless of future connection schemes. Similarly, the linking tool is simplified. In this case, a modal correction is necessary at the connected ports, but this correction is always the same and can be applied systematically.

\section{Cascading of two general devices}

In this section, the traditional approach for the cascade connection of two devices with an arbitrary and different number of ports is included, in order to facilitate the readability of the paper. To pose the most general situation possible, these devices will be connected through $C$ different ports. These ports have to be ordered so that they are the last ports of the scattering matrices of both devices. In fact, this restriction can be always accomplished, since the ports can be reordered if necessary [8]. In this case, we can divide the GSM of a given device, for example, device $n$, into four blocks

- $\underline{\boldsymbol{S}}_{\mathrm{FF}}^{(n)}$ which contains all the GSM elements that must be used to calculate the emergent mode weights at the free ports in response

Q4 to the incident waves at the free ports. This is a matrix of size $\left(A_{n}-C\right) N_{\text {mod }} \times\left(A_{n}-C\right) N_{\text {mod }}=F_{n} N_{\text {mod }} \times F_{n} N_{\text {mod }}$, where $A_{n}$ is the number ports of device $n, C$ is the number of connected ports, $F_{n}=A_{n}-C$ is the number of free ports and $N_{\text {mod }}$ is the number of modes considered at each port.

- $\underline{\underline{S}}_{\mathrm{FC}}^{(n)}$, of size $F_{n} N_{\text {mod }} \times C N_{\text {mod }}$, which contains all the GSM elements that must be used to calculate the emergent mode weights at the free ports in response to the incident waves at the connected ports.

$175-\underline{\boldsymbol{S}}_{\mathrm{CF}}^{(n)}$, of size $C N_{\text {mod }} \times F_{n} N_{\text {mod }}$, which contains all the GSM elements that must be used to calculate the emergent mode weights at the connected ports in response to the incident waves at the free ports.

- $\underline{\underline{S}}_{\mathrm{CC}}^{(n)}$, of size $C N_{\text {mod }} \times C N_{\text {mod, }}$, which contains all the GSM elements that must be used to calculate the emergent mode weights at the connected ports in response to the incident waves at the connected ports.

This means that the GSM of device $n$ could be written

$$
\underline{\underline{\boldsymbol{S}}}^{(n)}=\left(\begin{array}{cc}
c c \underline{\boldsymbol{S}}_{\mathrm{FF}}^{(n)} & \underline{\underline{\boldsymbol{S}}}_{\mathrm{FC}}^{(n)} \\
\underline{\boldsymbol{S}}_{\mathrm{CF}}^{(n)} & \underline{\underline{S}}_{\mathrm{CC}}^{(n)}
\end{array}\right)
$$

and can be used to find the emergent mode weights at every port, , or connected, $b_{\mathrm{C}}^{(n)}$, in response to an arbitrary incidence which is expressed in terms of the incident mode weights, $\underline{a}_{\mathrm{F}}^{(n)}$ and $\underline{a}_{\mathrm{C}}^{(n)}$

$$
\left(\begin{array}{c}
c \underline{b}_{\mathrm{F}}^{(n)} \\
\underline{b}_{\mathrm{C}}^{(n)}
\end{array}\right)=\left(\begin{array}{cc}
c c \underline{\underline{S}}_{\mathrm{FF}}^{(n)} & \underline{\underline{\boldsymbol{S}}}_{\mathrm{FC}}^{(n)} \\
\underline{\underline{S}}_{\mathrm{CF}}^{(n)} & \underline{\underline{S}}_{\mathrm{CC}}^{(n)}
\end{array}\right)\left(\begin{array}{c}
c \underline{a}_{\mathrm{F}}^{(n)} \\
\underline{a}_{\mathrm{C}}^{(n)}
\end{array}\right)
$$

For a different device, for example, the device $m$, we could organise the scattering parameters following the same criterion so that we can write its GSM as

$$
\underline{\underline{\boldsymbol{S}}}^{(m)}=\left(\begin{array}{cc}
c c \underline{\underline{S}}_{\mathrm{FF}}^{(m)} & \underline{\underline{S}}_{\mathrm{FC}}^{(m)} \\
\underline{\underline{\boldsymbol{S}}}_{\mathrm{CF}}^{(m)} & \underline{\underline{S}}_{\mathrm{CC}}^{(m)}
\end{array}\right)
$$

$$
\left(\begin{array}{c}
c \underline{b}_{\mathrm{F}}^{(m)} \\
\underline{b}_{\mathrm{C}}^{(m)}
\end{array}\right)=\left(\begin{array}{cc}
c c \underline{\boldsymbol{S}}_{\mathrm{FF}}^{(m)} & \underline{\underline{\boldsymbol{S}}}_{\mathrm{FC}}^{(m)} \\
\underline{\underline{\boldsymbol{S}}}_{\mathrm{CF}}^{(m)} & \underline{\underline{\boldsymbol{S}}}_{\mathrm{CC}}^{(m)}
\end{array}\right)\left(\begin{array}{c}
c \underline{a}_{\mathrm{F}}^{(m)} \\
\underline{a}_{\mathrm{C}}^{(m)}
\end{array}\right)
$$

Since we have cascaded both devices, we can write

$$
\begin{gathered}
\underline{b}_{\mathrm{F}}^{(n)}=\underline{\underline{\boldsymbol{S}}}_{\mathrm{FF}}^{(n)} \underline{a}_{\mathrm{F}}^{(n)}+\underline{\underline{\boldsymbol{S}}}_{\mathrm{FC}}^{(n)} \underline{a}_{\mathrm{C}}^{(n)} \\
\underline{b}_{\mathrm{C}}^{(n)}=\underline{\underline{S}}_{\mathrm{CF}}^{(n)} \underline{a}_{\mathrm{F}}^{(n)}+\underline{\boldsymbol{S}}_{\mathrm{CC}}^{(n)} \underline{a}_{\mathrm{C}}^{(n)} \\
\underline{b}_{\mathrm{F}}^{(m)}=\underline{\underline{\boldsymbol{S}}}_{\mathrm{FF}}^{(m)} \underline{a}_{\mathrm{F}}^{(m)}+\underline{\underline{\boldsymbol{S}}}_{\mathrm{FC}}^{(m)} \underline{a}_{\mathrm{C}}^{(m)} \\
\underline{b}_{\mathrm{C}}^{(m)}=\underline{\underline{S}}_{\mathrm{CF}}^{(m)} \underline{\mathrm{a}}_{\mathrm{F}}^{(m)}+\underline{\underline{S}}_{\mathrm{CC}}^{(m)} \underline{a}_{\mathrm{C}}^{(m)} \\
\underline{a}_{\mathrm{C}}^{(n)}=\underline{b}_{\mathrm{C}}^{(m)} \\
\underline{a}_{\mathrm{C}}^{(m)}=\underline{b}_{\mathrm{C}}^{(n)}
\end{gathered}
$$

In this case, in order to solve the connection, the modes emerging from the connected ports of the second device are equalled to the incident modes of the first device (9) and viceversa (10), as usual. By operating this set of equations one can obtain the global GSM of the connection

$$
\underline{\underline{\boldsymbol{S}}}=\left(\begin{array}{ll}
c c \underline{\underline{\boldsymbol{S}}}_{\mathrm{FF}}^{(n n)} & \underline{\underline{S}}_{\mathrm{FF}}^{(n m)} \\
\underline{\underline{\boldsymbol{S}}}_{\mathrm{FF}}^{(m n)} & \underline{\underline{S}}_{\mathrm{FF}}^{(m m)}
\end{array}\right)
$$

where

$$
\begin{gathered}
\underline{\underline{\boldsymbol{S}}}_{\mathrm{FF}}^{(n n)}=\underline{\underline{\boldsymbol{S}}}_{\mathrm{FF}}^{(n)}+\underline{\underline{\boldsymbol{S}}}_{\mathrm{FC}}^{(n)} \underline{\underline{\boldsymbol{S}}}_{\mathrm{CC}}^{(m)} \underline{\underline{F}}_{1} \underline{\underline{S}}_{\mathrm{CF}}^{(n)} \\
\underline{\underline{\boldsymbol{S}}}_{\mathrm{FF}}^{(n m)}=\underline{\underline{\boldsymbol{S}}}_{\mathrm{FC}}^{(n)} \underline{\underline{F}}_{2} \underline{\underline{\boldsymbol{S}}}_{\mathrm{CF}}^{(m)} \\
\underline{\underline{\boldsymbol{S}}}_{\mathrm{FF}}^{(m n)}=\underline{\underline{\boldsymbol{S}}}_{\mathrm{FC}}^{(m)} \underline{\underline{F}}_{1} \underline{\underline{\boldsymbol{S}}}_{\mathrm{CF}}^{(n)} \\
\underline{\underline{\boldsymbol{S}}}_{\mathrm{FF}}^{(m m)}=\underline{\underline{\boldsymbol{S}}}_{\mathrm{FF}}^{(m)}+\underline{\underline{\boldsymbol{S}}}_{\mathrm{FC}}^{(m)} \underline{\underline{S}}_{\mathrm{CC}}^{(n)} \underline{F}_{2} \underline{\underline{S}}_{\mathrm{CF}}^{(m)}
\end{gathered}
$$

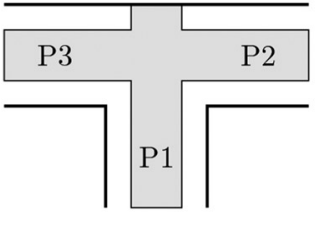

a
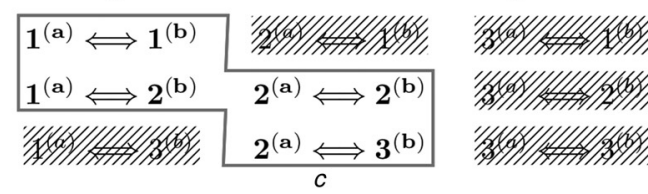

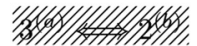

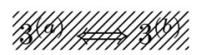

Fig. 1 Dimensions and port arrangement for the individual T-junctions $a$ Port numbering; top view

$b$ Dimensions $\left(a=19.05 \mathrm{~mm}, b=a / 2, a_{\mathrm{r}}=a / 2\right.$ and $\left.b_{\mathrm{r}}=a / 4\right)$ of the three-port example network: a single ridge rectangular waveguide $H$-plane T-junction; port view $c$ Possible connections of two different three-port networks; possible connections For the connection of two identical T-junctions, only the highlighted possibilities actually provide different networks 
Fig. 2 Possible networks obtained from the cascading of two identical H-plane T-junctions (19.05 $\mathrm{mm}$ width) in rectangular waveguide with final port-numbering

$a 1^{(a)} \Leftrightarrow 2^{(b)}$

$b 2^{(a)} \Leftrightarrow 3^{(b)}$

$c 1^{(a)} \Leftrightarrow 1^{(b)}$

$d 2^{(a)} \Leftrightarrow 2^{(b)}$

with

290

$$
\begin{aligned}
& \underline{\underline{F}}_{1}=\left(\underline{\underline{I}}-\underline{\underline{S}}_{\mathrm{CC}}^{(n)} \underline{\underline{S}}_{\mathrm{CC}}^{(m)}\right)^{-1} \\
& \underline{\underline{F}}_{2}=\left(\underline{\underline{I}}-\underline{\underline{\boldsymbol{S}}}_{\mathrm{CC}}^{(m)} \underline{\underline{S C}}_{\mathrm{CC}}^{(n)}\right)^{-1}
\end{aligned}
$$

295

These expressions (or equivalent expressions [9]) can be easily found in the microwave literature, for example in [1, 2, 5, 6, 8-13].

\section{Cascading of two simple three-port devices with feeding lines showing a single symmetry}

In this section, the problems regarding the cascading of multiport devices with feeding lines showing a single symmetry will be studied. Specifically, in order to discuss the problems concerning the reutilisation of GSMs when the traditional expressions of (12) (15) are applied, the different possible cascades of two identical single ridge rectangular waveguide $H$-plane T-junctions [14] will be analysed. The dimensions and port arrangement for the individual T-junctions are highlighted in Figs. $1 a$ and $b$.

There are nine possible self-cascades for this three-port device. All these possible connections can be seen in Fig. 1c. However, since the T-junction is symmetric, only four different networks can be actually obtained (see again Fig. 1c). The port numbering of the different networks resulting from the cascade connection of two identical T-junctions can be seen in Fig. 2.

The final port-numbering preserves the original ordering of the ports. For example, in the network of Fig. $2 a$, the first port of the first T-junction is connected, so that its second port becomes the first port of the final network and its third port, the second one. Since the second port of the second network is connected, its first port becomes the third port of the global network and its third port, the fourth one.

To obtain an individual, and unique for both networks, GSM, which could be applied to solve the connections of Figs. $2 a$ and $b$, the port reference systems, or the modal polarity, must be properly defined. The individual T-junction has been characterised with the commercial software Ansys HFSS ${ }^{\mathrm{TM}}$, so that Fig. 3 shows three possible sets of integration lines that ensure a correct connection of port 1 with port 2 , and port 2 with port 3 .

The results of the cascade connections shown in Figs. $2 a$ and $b$ are compared with a direct analysis of the resulting four-port networks with Ansys $\mathrm{HFSS}^{\mathrm{TM}}$. As expected, both results are virtually identical (not shown here).

Unfortunately, it is impossible to define a coincident set of integration lines to link port 1 with itself, or port 2 with itself (Figs. $2 c$ and $d$ ). In Fig. $4 a$, one can see the comparison of the direct and cascaded scattering parameters for the network of Fig. $2 c$, which have been calculated with $\mathrm{HFSS}^{\mathrm{TM}}$. In this case, the results are slightly different. The response provided by the cascade throws a global GSM with ports 3 and 4 interchanged. This is due to the fact that the equality imposed by (9) and (10) is not strictly true, because of the difference between the reference systems of the ports. Since the modes with odd symmetry are actually inverted, the lower T-junction is reflected and that is the reason why its ports (ports 3 and 4 in the global network) are interchanged.
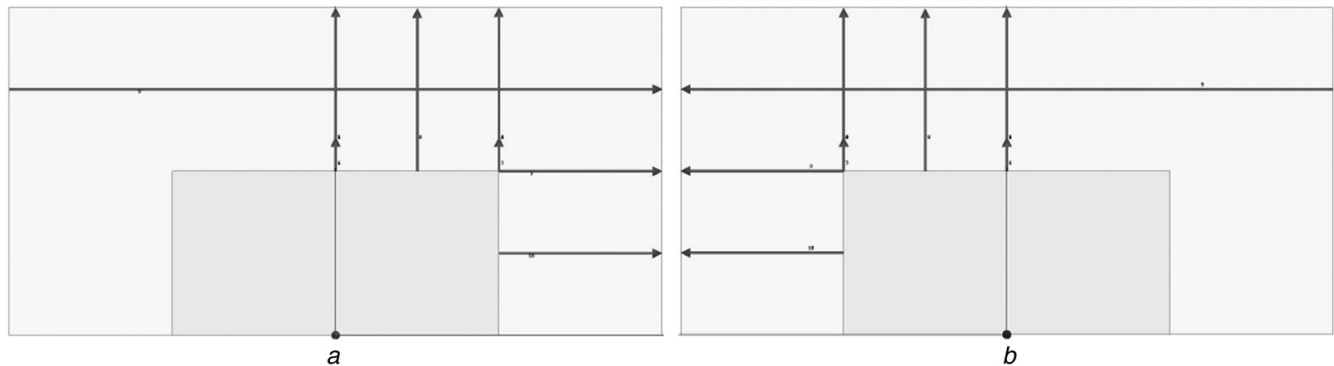

Fig. 3 Integration line sets (ten modes) defined to analyse the cascade of two identical single ridge rectangular waveguide H-plane T-junctions with Ansys $\operatorname{HFSS}^{\mathrm{TM}}$

$a$ Port 1 integration lines

$b$ Port 2 integration lines

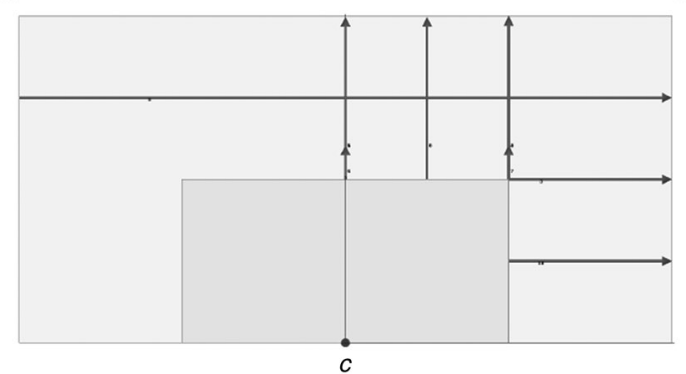



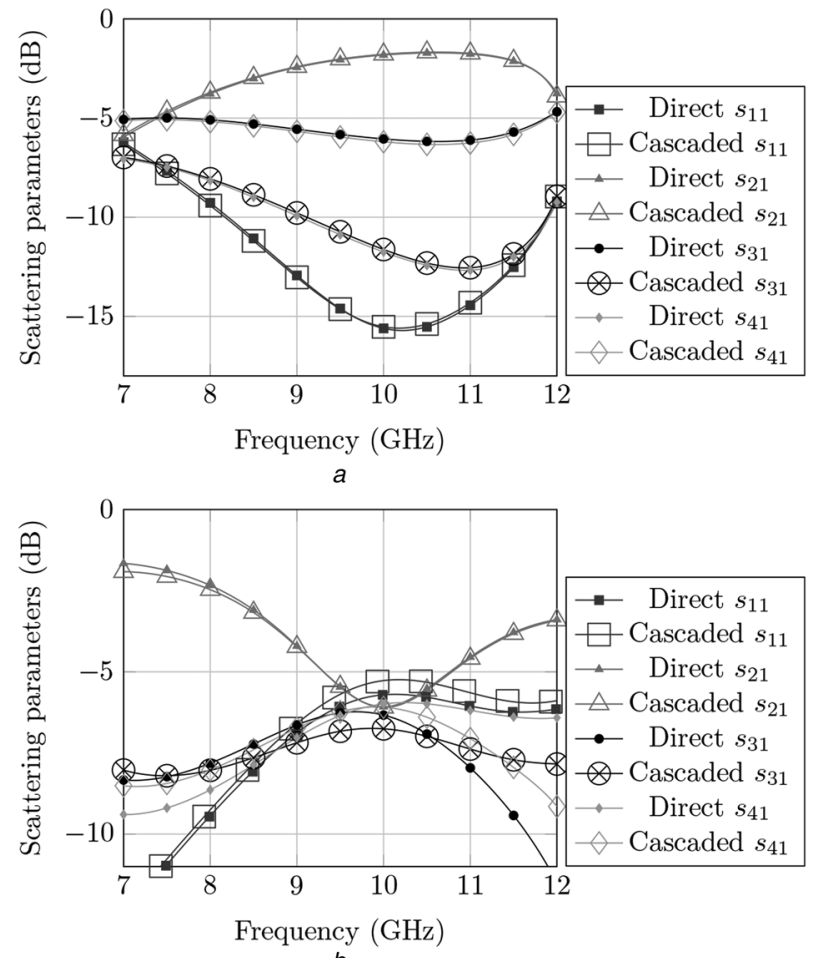

Fig. 4 Scattering parameters for the network of Figs. $2 c$ and $d$ (T-junction for a ridge rectangular waveguide)

Results labelled as 'Direct' have been calculated by directly solving the whole four-port network with Ansys HFSS ${ }^{\mathrm{TM}}$. Results labelled as 'Cascaded' have been obtained by cascading two identical T-junction GSMs using the traditional expressions of (12)-(15) $a$ Fig. $2 c$

$b$ Fig. $2 d$

\section{Cascading of two simple three-port devices with feeding lines showing a double symmetry}

To analyse this problem, the cascade of two identical $H$-plane rectangular waveguide T-junctions will be studied (see Fig. 5).

There are 18 possible self-cascades for this T-junction, while only 9 for a T-junction with ridge (see Fig. 1c). In this case, every port can be connected directly, or rotated, since the symmetry is double, and this is the reason why the possible connections are multiplied by 2. Anyway, actually, only four different networks can be obtained from the cascading of two identical versions of this device (see again Fig. 1c).

This device has been analysed, again, using the commercial software Ansys HFSS ${ }^{\mathrm{TM}}$. However, the modes of a rectangular waveguide can be solved analytically. Therefore in this case, the

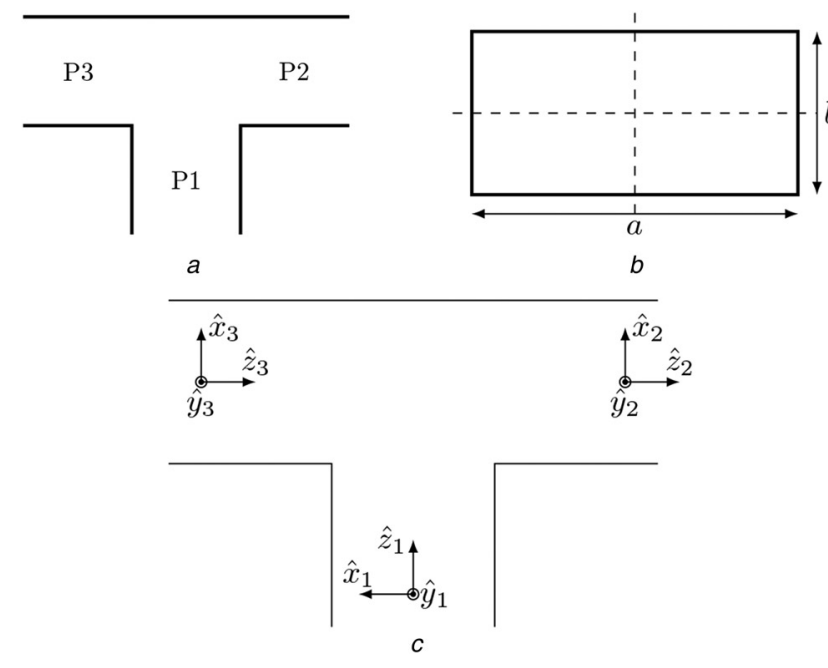

465

Fig. 5 Cascade of two identical H-plane rectangular waveguide T-junctions

$a$ Port numbering for a rectangular waveguide $H$-plane T-junction; top view

$b$ Dimensions $(a=22.86 \mathrm{~mm}, b=10.16 \mathrm{~mm})$ of the three-port example network with doubly symmetric feeding lines: a rectangular waveguide $i$-plane T-junction; port view $c$ Local port reference systems defined to analyse the cascade of two identical rectangular waveguide $H$-plane T-junctions with Ansys $\mathrm{HFSS}^{\mathrm{TM}}$; reference systems at the ports

polarities of these modes can be fixed by selecting an appropriate local reference system for each port in $\mathrm{HFSS}^{\mathrm{TM}}$ (the integration lines are not necessary with rectangular waveguide ports). As for the T-junction with ridge, the reference system will be selected so that port 1 could be connected directly, that is, without rotating the device, to port 2 , or port 2 to port 3 (see Fig. $5 c$ ).

As it also occurs in the previous section, the results of the direct analysis of the connections of Fig. 2 (for the $H$-plane T-junction in standard rectangular waveguide) are identical to the results of the equivalent cascades (not shown here). However, again, the cascades involving the connection of a port with itself cannot be solved unless a modal correction is applied. In fact, if the cascade connection for the network of Fig. $2 d$ is compared with the direct solution, the results notably differ (see Fig. 6).

\section{Modified cascading of two general devices with symmetrical feeding lines}

In this section, a coherent set of reference systems (or modal integration lines) is proposed so that the modal correction is always necessary but, at the same time, is always the same. Since

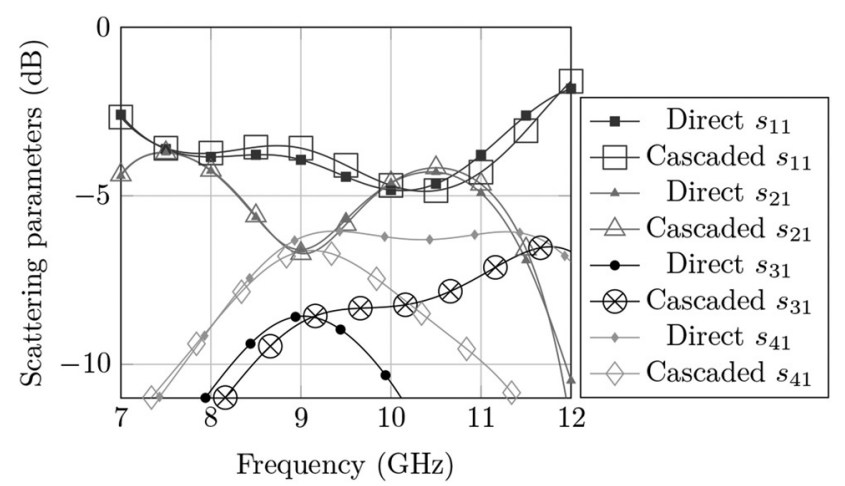

Fig. 6 Scattering parameters for the network of Fig. $2 d$ (T-junction for a standard rectangular waveguide)

Results labelled as 'Direct' have been calculated by directly solving the whole four-port network with Ansys HFSS ${ }^{\mathrm{TM}}$. Results labelled as 'Cascaded' have been obtained by cascading two identical T-junction GSMs using the traditional expressions of (12)-(15) 

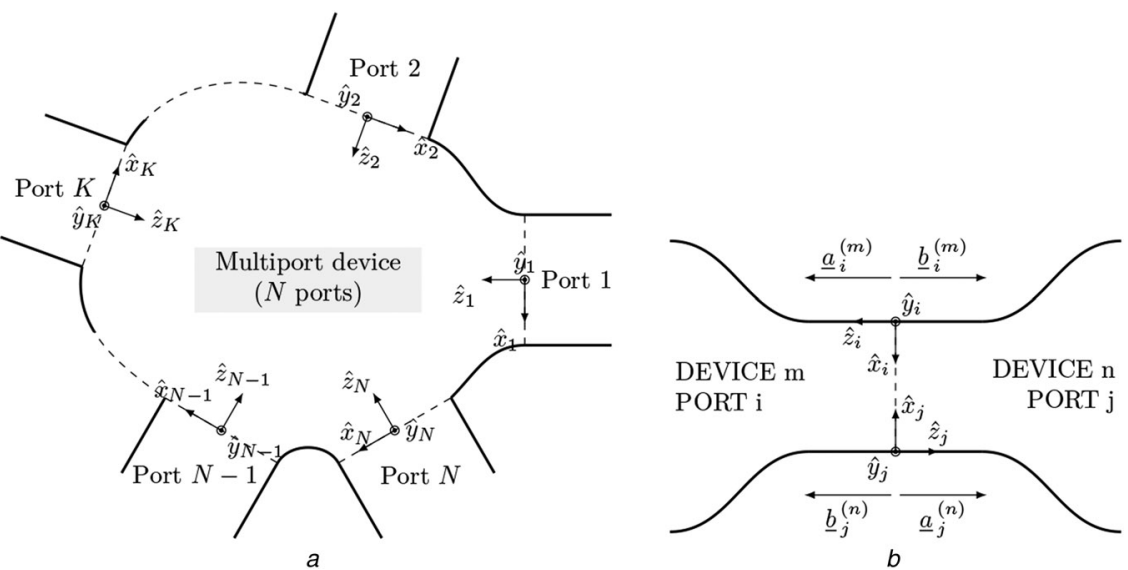

Fig. 7 Reflected waves and regressive waves

$a$ Multiport device with symmetric feeding lines and consistent port reference systems $b$ Connection of two ports and their reference systems the correction is always present and it is uniform, it can be incorporated to the traditional expressions of (12)-(15). Therefore freely connected, without worrying anymore about being continuously forced to check the appropriate polarity of the modes.

The proposed port reference systems are chosen so that the incident waves are formulated in terms of progressive waves

555 (towards $-\hat{z}$ propagation), whereas the reflected waves are formulated in terms of regressive waves (see Fig. 7a). If the ports cannot be solved analytically, then integration lines (or other means of defining modal polarity) are necessary. In this case, the integration lines associated to the different modes considered the device. For example, if the integration line associated to the second mode of the port is placed halfway in the right side of the port, and pointing upwards, then it should be defined exactly this way in every port of the device.

If the port reference systems (or polarities) are defined as explained before, (5)-(10) can be rewritten as follows

$$
\begin{gathered}
\underline{b}_{\mathrm{F}}^{(n)}=\underline{\underline{\boldsymbol{S}}}_{\mathrm{FF}}^{(n)} \underline{a}_{\mathrm{F}}^{(n)}+\underline{\underline{\boldsymbol{S}}}_{\mathrm{FC}}^{(n)} \underline{a}_{\mathrm{C}}^{(n)} \\
\underline{b}_{\mathrm{C}}^{(n)}=\underline{\underline{\boldsymbol{S}}}_{\mathrm{CF}}^{(n)} \underline{a}_{\mathrm{F}}^{(n)}+\underline{\underline{S}}_{\mathrm{CC}}^{(n)} \underline{a}_{\mathrm{C}}^{(n)} \\
\underline{b}_{\mathrm{F}}^{(m)}=\underline{\underline{S}}_{\mathrm{FF}}^{(m)} \underline{a}_{\mathrm{F}}^{(m)}+\underline{\underline{S}}_{\mathrm{FC}}^{(m)} \underline{\mathrm{a}}_{\mathrm{C}}^{(m)} \\
\underline{b}_{\mathrm{C}}^{(m)}=\underline{\underline{S}}_{\mathrm{CF}}^{(m)} \underline{a}_{\mathrm{F}}^{(m)}+\underline{\underline{\boldsymbol{S}}}_{\mathrm{CC}}^{(m)} \underline{\mathrm{a}}_{\mathrm{C}}^{(m)}
\end{gathered}
$$

$$
\begin{aligned}
& \underline{a}_{\mathrm{C}}^{(n)}=\underline{\underline{\boldsymbol{D}}}_{\mathrm{C}}^{(n)} \underline{b}_{\mathrm{C}}^{((m)} \\
& \underline{\boldsymbol{a}}_{\mathrm{C}}^{(m)}=\underline{\underline{\boldsymbol{D}}}_{\mathrm{C}}^{(m)} \underline{\boldsymbol{b}}_{\mathrm{C}}^{(n)}
\end{aligned}
$$

580

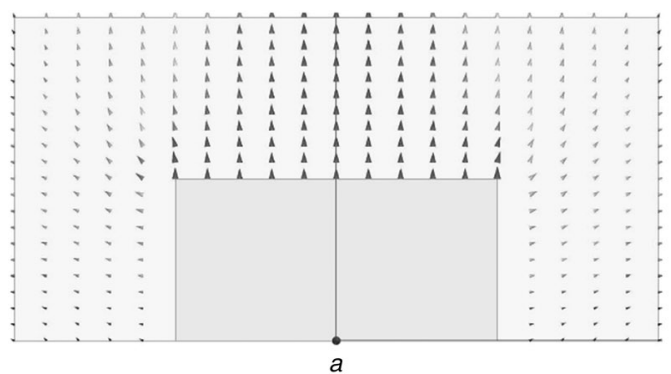
a general cascading procedure is obtained and the ports can be
With these coherent reference systems, the reflected waves at the connected ports of device $n$ cannot be directly equal to the incident waves to the connected ports of device $m$. Similarly, the reflected waves at the connected ports of device $m$ are not equal to the incident waves to the ports of device $n$.

The modes of a waveguide that present a single symmetry will show the same kind of symmetry (see Fig. 7b). Therefore if the transversal field pattern of one of these modes is folded along its symmetry axis, both superimposed field patterns will be, either strictly identical, that is, the field pattern of the selected mode shows a bilateral symmetry (see Fig. $8 a$ ), or identical in modulus but opposite in sense, that is, the field pattern shows an inverted bilateral symmetry (see Fig. $8 b$ ). When both halves strictly match, the modes will be identical for both connected ports. In this case, the modal weights can be compared directly. On the other hand, when both halves show opposite sense, a sign change is necessary in order to exactly match the modes of the connected ports. Obviously, this sign change has to be considered. Therefore one of the modal sets has to be adjusted in (22) and (23), for example, the emergent modal weights, $\underline{b}_{C}^{(m)}$ and $\underline{b}_{C}^{(n)}$, by multiplying them by an appropriate diagonal matrix (i.e. $\underline{\underline{D}}_{C}^{(n)}$ and $\underline{\boldsymbol{D}}_{\mathrm{C}}^{(m)}$, respectively). The elements of this diagonal matrix will be 1 , if the corresponding modal field pattern shows bilateral symmetry, and -1 otherwise. A knowledge of the transversal field pattern of the modes is needed in order to discriminate which modes present bilateral symmetry.

If the waveguide shows a double symmetry, the situation is slightly more complex. In this case, the connection can be direct, that is, with coincident vertical axis $(\hat{y})$ and opposite horizontal axis $(\hat{x})$, as in Fig. $7 b$, or turned, that is, with coincident horizontal axis and opposite vertical axis. In the first situation, the elements of the diagonal matrices of (22) and (23), related to the modes showing horizontal bilateral symmetry (e.g. the modes shown in

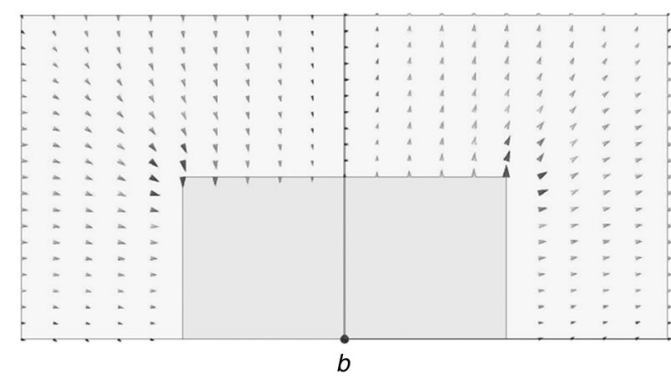

Fig. 8 Electric field for two different single ridge rectangular waveguide modes (HFSS ${ }^{\mathrm{TM}}$ )

$a$ Sign change not required

$b$ Sign change required

$a$ Mode with bilateral symmetry

$b$ Mode with inverted bilateral symmetry 


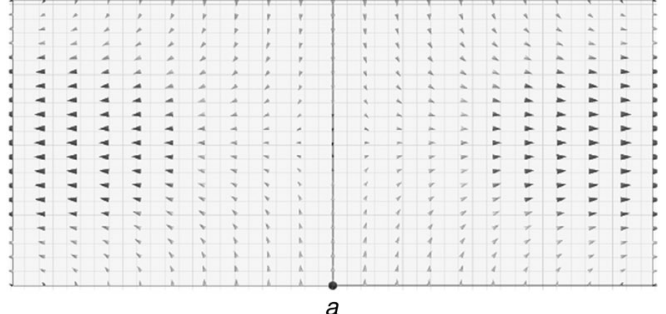

670

with

$$
\underline{\underline{\boldsymbol{S}}}_{\mathrm{FF}}^{(m m)}=\underline{\underline{\boldsymbol{S}}}_{\mathrm{FF}}^{(m)}+\underline{\underline{\boldsymbol{S}}}_{\mathrm{FC}}^{(m)} \underline{\underline{C}}_{\mathrm{C}}^{(m)} \underline{\underline{S}}_{\mathrm{CC}}^{(n)} \underline{\underline{\boldsymbol{D}}}_{\mathrm{C}}^{(n)} \underline{\underline{F}}_{2} \underline{\underline{\boldsymbol{S}}}_{\mathrm{CF}}^{(m)}
$$

From the programmer point of view, it is more convenient to apply the modal correction in the connection stage rather than in the solving stage, which implies selecting modified reference systems or modal polarities at the ports, depending on subsequent connection schemes. With this proposal, on one hand, an independent code can perform an individual analysis of every device without worrying about which ports are going to be connected and, on the other hand, during the linking stage, the recursive cascading-by-pairs piece of code can blindly apply the appropriate regular corrections at the connected ports, and then link the different building blocks.

Besides, this modal correction can be used to characterise other mismatches at the ports. Perhaps, the most useful mismatch to characterise could be a possible separation between connected ports, that is, ports are not directly connected, they are connected through a piece of empty waveguide of certain length. In this case, (22) and (23) must be reformulated.

$$
\begin{aligned}
& \underline{a}_{\mathrm{C}}^{(n)}=\underline{\underline{\boldsymbol{D}}}_{\mathrm{C} 2}^{(n)} \underline{\underline{D}}_{\mathrm{C} 1}^{(n)} \underline{b}_{\mathrm{C}}^{(m)} \\
& \underline{a}_{\mathrm{C}}^{(m)}=\underline{\boldsymbol{D}}_{\mathrm{C} 2}^{(n)} \underline{\underline{D}}_{\mathrm{C} 1}^{(m)} \underline{\mathrm{C}}_{\mathrm{C}}^{(n)}
\end{aligned}
$$
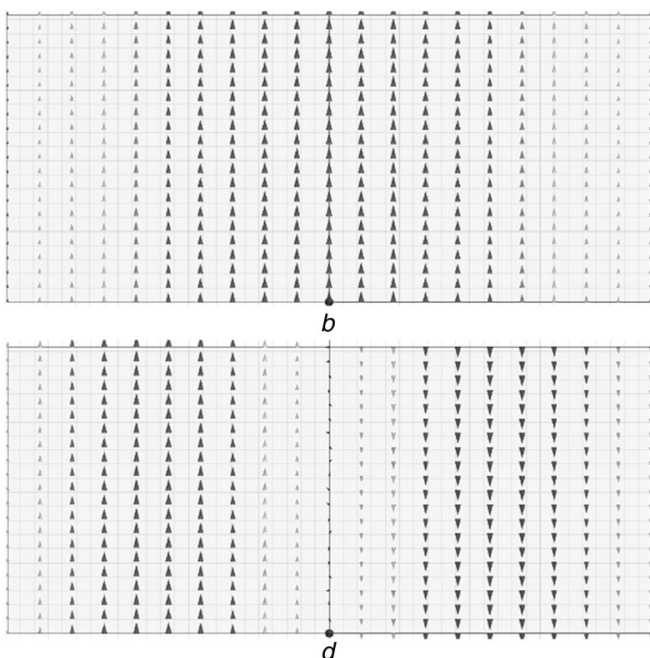

where $\underline{\boldsymbol{D}}_{\mathrm{Cl}}^{(i)}$ is the modal conversion explained in this section for $\mathbf{Q 5}$ device $\bar{i} ; \underline{D}_{\mathrm{C} 2}^{(i)}$ can be used to propagate the incident waves to device $i$ along a certain distance at every connected port.

It is widely known that these propagation matrices are also diagonal. This means that these new diagonal matrices can be combined with the old ones into single diagonal matrices.
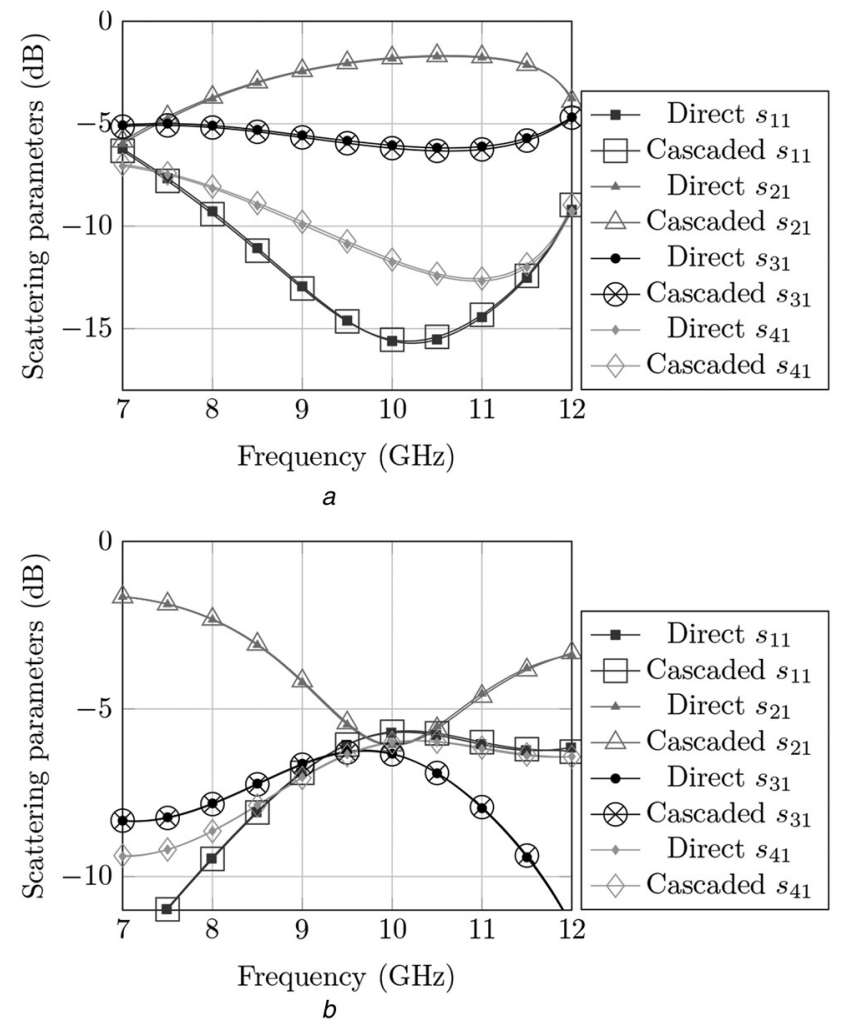

Fig. 10 Scattering parameters for the network of Figs. $2 c$ and d (T-junction for a ridge rectangular waveguide)

Results labelled as 'Direct' have been calculated by directly solving the whole four-port network with Ansys HFSS ${ }^{\mathrm{TM}}$. Results labelled as 'Cascaded' have been obtained by cascading two identical T-junction GSMs using the modified cascading procedure of this paper

$a$ Fig. $2 c$

$b$ Fig. $2 d$ 


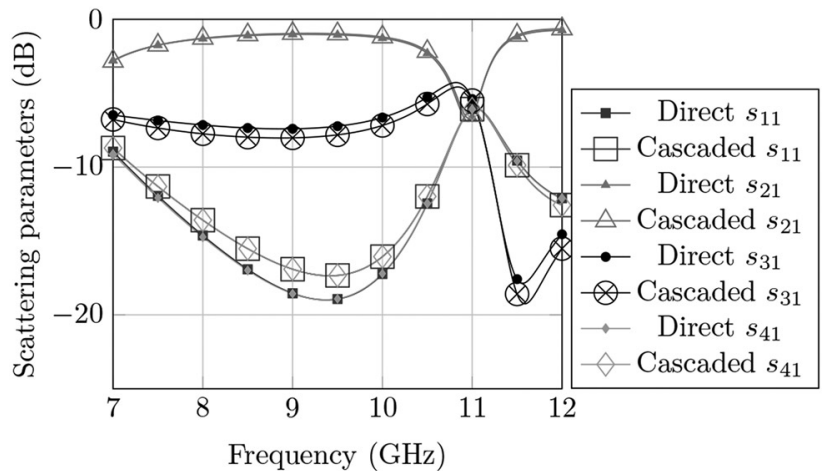

a

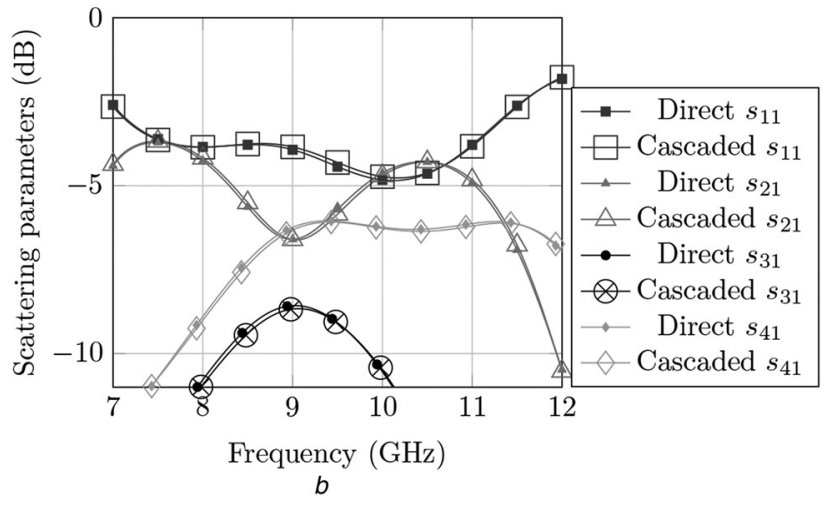

820

Fig. 11 Scattering parameters for the network of Figs. 2c and d (T-junction for a standard rectangular waveguide and direct connection)

Results labelled as 'Direct' have been calculated by directly solving the whole four-port network with Ansys HFSS ${ }^{\mathrm{TM}}$. Results labelled as 'Cascaded' have been obtained by cascading two identical T-junction GSMs using the modified cascading procedure of 825 this paper

Proceeding in this way, the original expressions for (22) and (23) are 830 recovered

$$
\begin{aligned}
& \underline{a}_{\mathrm{C}}^{(n)}=\underline{\underline{D}}_{\mathrm{C}}^{(n)} \underline{b}_{\mathrm{C}}^{(m)} \\
& \underline{a}_{\mathrm{C}}^{(m)}=\underline{\underline{\boldsymbol{D}}}_{\mathrm{C}}^{(m)} \underline{b}_{\mathrm{C}}^{(n)}
\end{aligned}
$$

so that the expressions in this section are valid even when the ports are not directly connected.

When the ports are connected through a piece of empty waveguide, this fact is usually characterised by treating the empty

840 waveguide as an additional network to be cascaded. This strategy notably increases the cost of the cascading; on the contrary, the correction proposed in this paper can be achieved at a low cost (a few products by diagonal matrices).

\section{Results}

In this section, the modified cascading procedure has been applied to solve the cascaded networks of Fig. 2. Figs. $10 a$ and $b$ show the comparison of the results provided by the modified cascading

850 procedure presented in this paper, and the direct solution of the problematic networks of Figs. $2 c$ and $2 d$, respectively, for the ridge rectangular waveguide.

The cascade behaves as expected, since there is an almost exact match of the direct and cascaded solutions. Besides, the cascading 855 procedure is completely general. In fact, all of the combinations shown in Fig. $1 c$ have been successfully tested.

The connection of standard rectangular waveguide T-junctions has been solved as well. Results can be seen in Figs. $11 a$ and $b$

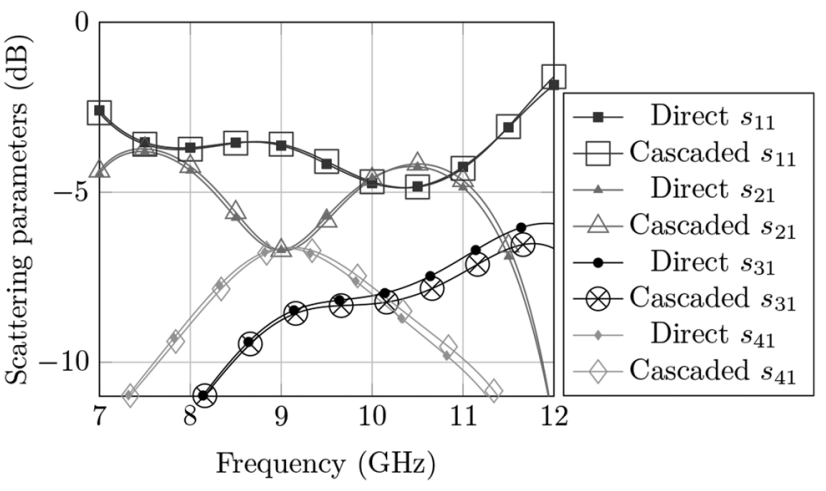

860

Fig. 12 Scattering parameters when port 2 is connected to itself, but turned (T-junction for a standard rectangular waveguide)

Results labelled as 'Direct' have been calculated by directly solving the whole four-port network with Ansys HFSS ${ }^{\mathrm{TM}}$. Results labelled as 'Cascaded' have been obtained by cascading two identical T-junction GSMs using the modified cascading procedure of this paper

for a direct connection of the ports. In this case, both problematic networks can be appropriately solved.

When port 2 is connected with itself, but turned, the resulting network is the one in Fig. $2 b$, instead of the one in Fig. $2 d$. In this case, the modal correction must be applied to the modes with inverted vertical bilateral symmetry. The results recovered after applying this correction have been compared with the direct analysis of the network of Fig. $2 b$, and can be seen in Fig. 12.

The expected response has been recovered so it can be concluded that the proposed modal correction is even valid for doubly symmetric feeding waveguides.

\section{Conclusion}

In this paper, a coherent definition for the mode polarity, or the reference systems of the ports, is proposed. In the building-blocks solving stage, all of the ports are characterised in the same manner, regardless if they are going to be connected or not. Finally, in the cascading stage, the modified expressions of (24)(29) can be used to obtain the global GSM, by applying, for example, a cascading-by-pairs recursive scheme. Therefore with this approach, the reutilisation of previously calculated GSMs is trivial. Besides, it is appropriate for characterising, without increasing the solving cost, the connection of ports separated by a certain physical distance.

\section{Acknowledgment}

This work was supported by the Ministry of Science and Innovation, Spanish Government, under Research Projects TEC2013-47037C05-3-R and TEC2013-47037-C05-1-R.

\section{References}

Pozar, D.M.: 'Microwave engineering' (John Wiley \& Sons, Inc., Hoboken, New Jersey, 2005, 2nd edn.)

2 Budimir, D.: 'Generalized filter design by computer optimization' (Artech House, London, 1998)

3 Redheffer, R.: 'Inequalities for a matrix Riccati equation', J. Math. Mech., 1959, 8, (3), pp. 349-367

4 Stock, D., Kaplan, L.: 'A comment on the scattering matrix of cascaded 2n-ports (correspondence)', IRE Trans. Microw. Theory Tech., 1961, 9, (5), pp. 454-454

5 Kaplan, L., Stock, D.: 'A generalization of the matrix Riccati equation and the 'star' multiplication of Redheffer', J. Math. Mech., 1962, 11, (6), pp. 927-928

6 Chu, T.S., Itoh, T.: 'Generalized scattering matrix method for analysis of cascaded and offset microstrip step discontinuities', IEEE Trans. Microw. Theory Tech. 1986, 34, (2), pp. 280-284

7 Belenguer, A., Diaz Caballero, E., Esteban, H., Borja, A.L., Cascon, J.: 'Krylov's solver based technique for the cascade connection of multiple $n$-port multimodal scattering matrices', IEEE Trans. Microw. Theory Tech., 2013, 61, (2), pp. 720-726 

Symp. Dig., 1981, 81, (1), pp. 507-509

9 Overfelt, P., White, D.: 'Alternate forms of the generalized composite scattering matrix', IEEE Trans. Microw. Theory Tech., 1989, 37, (8), pp. 1267-1268

10 James, G.: 'Analysis and design of TE11-to-HE11 corrugated cylindrical waveguide mode converters', IEEE Trans. Microw. Theory Tech., 1981, 29, (10), pp. 1059-1066

11 Wan, C., Encinar, J.: 'Efficient computation of generalized scattering matrix for analyzing multilayered periodic structures', IEEE Trans. Antennas Propag., 1995, 43, (11), pp. 1233-1242
12 Jones, R., Baboi, N., Tantawi, S., Kroll, N.: 'Circuit and scattering matrix analysis of the wire measurement method of beam impedance in accelerating structures'. Proc. of the Particle Accelerator Conf., 2003, PAC 2003, May 2003, vol. 2, pp. $1270-1272$

13 Bachiller, C., Esteban, H., Boria, V.E., Belenguer, A., Morro, J.V.: 'Efficient technique for the cascade connection of multiple two-port scattering matrices', IEEE Trans. Microw. Theory Tech., 2007, 55, (9), pp. 1880-1886

14 Marcuvitz, N.: 'Waveguide handbook', in (Ed.): 'IEE Electromagnetic Wave Series 21' (London, 1986) 


\section{MAP20140167}

Author Queries

1060 Angel Belenguer, Alejandro Lucas Borja, Elena Diaz Caballero, Hector Esteban, Vicente E. Boria

Q1 Please confirm the given affiliations to the authors.

Q2 Abstract text should not exceed 200 words as per style. Please check and change.

Q3 Please confirm the change made from 'not symmetric' to 'non-symmetric'.

Q4 IET style for matrices and vectors is to use bold italics. Please check that we have identified all instances.

Q5 Please check $\underline{\underline{D}}_{\mathrm{C} 1}^{(i)}$ and $\underline{\underline{D}}_{\mathrm{C} 2}^{(i)}$ are not used in equation but explanation is given.

Q6 Please provide editor name and publisher name for Ref. [14].

Q7 We have inserted a main caption for Figs. (1, 5 and 7). Please check and confirm, else provide the same. 